\title{
细节特征保持的三维面部表情迁移方法
}

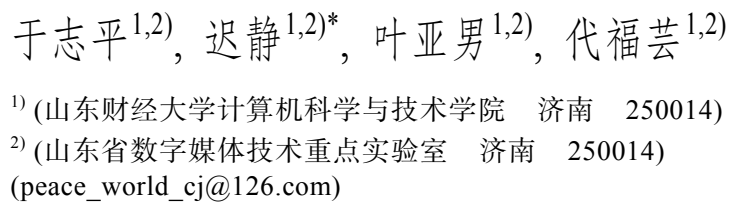

摘 要: 在三维面部表情迁移中, 针对保持目标模型丰富的细节信息以使生成的新表情真实自然, 以及减少表情迁 移的学习训练时间这 2 个热点问题, 提出一种细节特征保持的三维面部表情迁移方法. 首先提取三维面部模型的细 节特征，获得滤掉细节后的基本表情; 然后利用改进的有参无监督回归方法将源模型的基本表情传递给目标模型; 最后利用提出的细节特征向量调整策略对具有源基本表情的目标模型进行细节恢复. 在 Windows 10 系统的 Matlab 中, 以重建精度和训练时间为评价指标, 对 COMA 等三维面部数据集进行视觉对比和定量分析实验. 结果表明, 与 非线性联合学习方法相比，该方法在将源模型的表情无损迁移到目标模型的同时，很好地保持了目标模型自身的个 性细节特征，使生成的表情真实自然; 有效地提高了面部表情迁移的训练速度.

关键词：表情迁移；有参无监督回归；细节特征提取；细节特征恢复

中图法分类号: TP391.41 DOI: 10.3724/SP.J.1089.2021.18298

\section{Detailed Features-Preserving 3D Facial Expression Transfer}

\author{
Yu Zhiping ${ }^{1,2)}$, Chi Jing ${ }^{1,2)^{*}}$, Ye Yanan ${ }^{1,2)}$, and Dai Fuyun ${ }^{1,2)}$ \\ 1) (School of Computer Science and Technology, Shandong University of Finance and Economics, Jinan 250014) \\ 2) (Shandong Provincial Key Laboratory of Digital Media Technology, Jinan 250014)
}

\begin{abstract}
In the 3D facial expression transfer field, aiming at the two hot problems of preserving the rich detailed information of the target model to make the generated new expressions realistic and natural, and reducing the training time, this paper presents a new detailed features-preserving 3D facial expression transfer method. Firstly, the detailed features are extracted from 3D face models to obtain the basic expression models without details. Then, the basic expression of source model is transferred to the target model with the improved parametric dimensionality reduction by unsupervised regression. Finally, the detailed features of the target model are restored by using the proposed detailed feature vector adjustment strategy. The visual contrast and quantitative analysis experiments with reconstruction accuracy and training time as the evaluation indexes are conducted on the 3D facial datasets such as COMA in the Matlab software under the Windows 10 environment. The results illustrate that compared with the nonlinear co-learning method, the method can not only transfer the expression of the source model to the target model without losses, but also well preserve the personalized detailed features of the target model, so it can make the generated expressions more realistic and natural. The method also effectively improves the training speed in facial expression transfer.
\end{abstract}

收稿日期：2020-04-03; 修回日期：2020-08-18，基金项目：国家自然科学基金(61772309); 山东省省属优青项目(ZR2018JL022); 山东省重点研发计划(2019GSF109112); 山东省高等学校青创科技支持计划(2020KJN007); 山东省自然科学基金(ZR2019MF016); 山 东省高等学校优势学科人才团队培育计划. 于志平(1995一), 男, 硕士研究生, 主要研究方向为表情动画、数字几何处理; 迟静(1980一), 女, 博士, 教授, 博士生导师, 论文通讯作者, 主要研究方向为计算机动画、几何造型、医学图像处理; 叶亚男(1994一), 女, 硕士研 究生, 主要研究方向为计算机动画、数字图像处理; 代福芸(1996一), 女, 硕士研究生, 主要研究方向为表情动画. 
Key words: expression transfer; parametric dimensionality reduction by unsupervised regression; detailed features extraction; detailed features recovery

近年来，三维表情动画在计算机动画、影视特 效和虚拟现实等领域得到了广泛应用. 但是，由于 人类面部的生理结构极其复杂, 计算机在模拟面 部表情细节变化时, 往往会导致不自然表情的产 生. 因此, 如何在三维面部模型上重现高度真实感 的表情变化, 已成为计算机图形学领域最富有挑 战性的研究问题之一. 面部表情迁移技术可将已 有角色模型的表情动画复制到新的三维面部模型 上，有效地避免了为新模型制作表情动画序列的 烦琐工作，大大提高了现有动画的复用率和新动 画的合成效率, 为真实感表情动画的生成提供了 新的途径和方法, 已成为目前面部表情重现的研 究热点.

面部表情迁移方法需要满足以下要求: (1) 表 情具有高度的真实感, 脸部未发生扭曲, 表情自 然，能够较好地保持面部细节; (2) “即进即出”, 新 表情的合成速度快，延迟时间短；(3) “一次设计， 重复使用”，表情迁移方法应具备将已有表情复制 到任何面部几何结构不同的新模型上的能力，尽 可能减少迁移中的人工干预；(4) 具有简单、易用 的操作平台, 较好的人机交互能力. 然而现有的表 情迁移方法在这几个方面都存在着一定的不足.

传统的表情迁移方法大致可分为基于物理模 型的三维面部表情重建和基于参数化的面部表情 迁移. 基于物理模型的三维面部表情重建方法 ${ }^{[1]}$ 是 对三维面部构建肌肉或伪肌肉模型，通过控制肌 肉模型的运动, 实现面部表情的改变. 这种方法往 往需要过多的人工干预, 并且难以精确模拟皮肤 细节. 基于参数化的面部表情迁移是通过跟踪源 模型面部表情特征点的变化, 调节面部形态参数, 进而控制目标模型的表情变化. Dutreve 等 ${ }^{[2]}$ 利用 径向基函数(radial basis function, RBF)插值将源表 情特征点的偏移量重定向到目标模型, 为目标模 型创建面部表情. Chi 等 ${ }^{[3]}$ 利用顶点的时空相关性 自动对面部模型进行区域划分，并结合用户约束 和表情样本估算混合权重进行分区域建模，再将 不同区域进行传播融合，构建出新的表情模型. Chuang 等 ${ }^{[4]}$ 的双线性模型和 Vlasic 等 ${ }^{[5]}$ 的多线性 模型都能够实现表情迁移，然而人类面部表情处 于高维流形，线性模型难以细致地刻画面部表情 细节, 从而降低了目标表情的真实程度, 难以保证 目标表情具有较高的精度. Thies 等 ${ }^{[6]}$ 的方法实现
了视频层面的实时表情传递，它利用每帧图像的 身份、表情和皮肤反射率等信息构建参数模型，通 过计算参数空间中源表达式和目标表达式的差异 来修改目标参数. 为保证合成视频的真实感, 该方 法需要仔细处理光照和阴影, 过程极其耗费时间. Wan 等 ${ }^{[7]}$ 利用基于测地线距离的 RBF 插值方法, 克服了传统 RBF 方法引起的嘴部变形不自然的问 题, 实现了从二维视频到三维模型的表情传递. Feng 等 ${ }^{[8]}$ 利用典型关联分析方法从训练数据中学 习适合的变形子空间, 进而通过特征点的运动生 成模型局部区域变形，该方法适用于单模型表面 变形.

实现表情迁移的另一途径是基于机器学习的 方法, 通过对面部表情库中的样本进行统计分析, 提取面部表情的特征和影响因素来完成面部表情 的传递. Ma 等 ${ }^{[9]}$ 通过 CycleGAN 学习 Blendshape 空间中任意 2 个面部表情的映射, 之后利用构造的 三维面部模型、双向表情映射以及唇部矫正等信 息, 将源表情迁移到指定的目标人脸. Abrevaya 等 ${ }^{[10]}$ 将卷积神经网络中的编码器和基于多线性模型的 解码器相结合, 对三维面部模型进行解耦, 获得身 份属性和表情属性; 然后通过对 2 种属性进行解码 操作构建新的三维模型, 从而实现面部表情的改 变; 但其使用的多线性模型难以精确地反映处于 高维流形的面部表情. Liu 等 ${ }^{[11]}$ 引人解耦表示学习 的概念, 利用机器学习框架将面部模型进行属性 分解与合成, 以改变模型的面部表情. Jiang 等 ${ }^{[12]}$ 在解耦表示学习的基础上, 提出使用三维面部形 状空间来表示高维表情, 克服了线性模型的上述 弊端. 基于机器学习的表情迁移方法可以实现一 次设计, 重复使用, 但训练一个样本库往往需要付 出巨大的时间代价, 且目标面部表情的自然程度依 赖于所采用的机器学习方法和样本库的模型质量.

在表情迁移中, 三维几何模型的细节特征直 接影响着模型表情的真实感. 在模型发生大幅度 变形之后, 为了精确地再现物理模型的几何细节, 研究人员先后提出了多层次曲面变形 ${ }^{[13]}$ 和保细节 编辑 ${ }^{[14]}$ 等方法: (1) 多层次曲面变形是将原始空间 曲面进行分解，在获得一个光滑曲面和原曲面的 细节特征后, 对光滑曲面进行空间操作, 最后将细 节特征附加到变形后的光滑曲面上; 该方法对局 部几何细节的描述方式主要分为基于微分坐标表 
示和基于拉普拉斯坐标表示 2 种. (2) 保细节编辑 方法要求当几何模型的特征发生大尺度改变时, 模型的局部细节特征依然保持不变; 该方法对几 何细节的描述方式主要分为法向几何细节表示和 空间位置几何细节表示 2 种. Wan 等 ${ }^{[15]}$ 将二维视频 的表情迁移到三维模型时, 利用拉普拉斯变形将 特征点的运动传播到整个模型，从而保持了模型 的细节信息; 但该方法涉及求解大型线性系统，当 输人模型的顶点数量很大时, 其速度慢、占用内存 大. $\mathrm{Xu}$ 等 ${ }^{[16]}$ 将高保真度的三维模型分解为低分辨 率的光顺模型以及面部细节信息, 并分别处理这 2 部分信息, 实现了在完成表情迁移的同时, 保证目 标模型的细节信息.

针对现有方法中存在的各种问题，本文提出 一种细节特征保持的三维面部表情迁移方法. 该 方法不仅可以将源模型的面部表情迁移给目标模 型, 还可以使目标模型具有精细的表情细节, 且整 个过程不需要人工干预. 为使目标模型具有丰富 的细节和较高的真实感, 本文提出将表情迁移过 程分为基本表情迁移和表情细节恢复 2 部分的新 思路. 首先采用拉普拉斯光顺算法提取源模型和 目标模型的表情细节，得到滤掉细节后的基本表 情; 然后利用改进的有参无监督回归 (parametric dimensionality reduction by unsupervised regression, pDRUR)方法 ${ }^{[17]}$ 获得映射/重建函数, 实现基本表 情传递; 最后对经过基本表情迁移得到的具有源 表情的目标模型, 通过提出的调整规则优化其顶 点位置和法向, 完成对目标模型的面部表情细节 的恢复. 细节恢复后的目标模型不仅具有与源模 型一致的真实自然的表情, 而且保持了目标模型 自身的个性细节特征，如皱纹、褶皱和酒窝等，且 这些细节特征的变化与新表情相符.

\section{1 本文方法概述}

本文方法的输人为 2 组三维面部模型序列, 同 一组模型序列代表同一个人的各类表情集合, 且 序列内部各模型同构. 另外, 2 组模型序列中的部 分表情要求一一对应, 以满足联合训练阶段的需 要. 本文方法可实现表情在 2 组模型序列之间的任 意传递, 即一组模型上的表情可传递给另一组, 反 之亦然. 为方便表述, 本文将任意一组序列作为源 模型序列, 将该序列中的任意单个模型称为源模 型; 将另一组模型序列称为目标模型序列, 将该序 列中的任意单个模型称为目标模型. 本文方法的 输出是重建得到的具有源模型面部表情的目标模 型, 且重建的目标模型具有高度的真实感和丰富 的表情细节.

本文方法由如图 1 所示面部细节特征提取、联 合训练、源表情传递和目标模型细节特征恢复 4 部分构成. 在面部细节特征提取阶段对源模型序 列和目标模型序列应用拉普拉斯光顺算法, 获得 源/目标基本表情模型序列、源模型基本表情以及 目标模型细节特征向量, 分别用于联合训练、源表 情传递以及目标模型细节特征恢复. 其中, 基本表 情模型包含该人脸模型的基本表情信息, 面部细 节特征向量包含该模型精细的面部细节信息. 联 合训练阶段对源/目标基本表情模型序列应用函数 优化, 获得源/目标模型序列映射重建函数. 源表 情传递阶段利用源序列映射函数和目标序列重建 函数将源模型的基本表情传递给目标模型. 细节 恢复阶段利用目标模型面部细节特征向量, 进一 步调整目标模型上各顶点的位置和法向, 对具有 源表情的目标模型进行细节恢复.

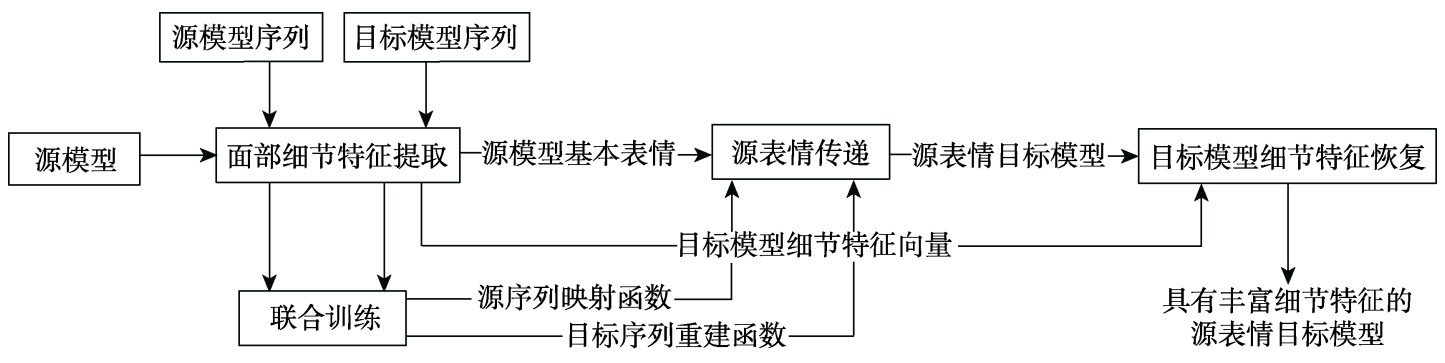

图 1 本文方法框架

\section{2 面部细节特征提取}

\section{1 基本思想}

三维面部模型通常由三角网格构成，网格上
的顶点位置直接决定着模型的基本表情和细节信 息. 其中, 基本表情反映面部的表情种类，包括微 笑、伤心和惊讶等常见表情; 细节信息一般指皮肤 皱纹等精细的特征, 这些细节往往使表情带有鲜 
明的个性化特征. 表情的变化反映到三角网格面 部模型上即模型顶点位置和法向的改变. 其中，基 本表情往往变化幅度比较大, 通常对应着较大的 顶点位置和法向的改变; 细节信息多是局部的微 小变形, 对应着较小的顶点位置和法向的改变.

本文对源/目标模型序列进行面部细节特征提 取，获得源/目标基本表情模型序列、源基本表情模 型和目标模型细节特征向量. 基本表情模型包含 面部模型的基本表情信息. 细节特征向量则包含 面部模型精细的表情细节信息，该信息能够精确 反映目标模型的特有属性，通过将该信息单独提 取并恢复到改变表情后的目标模型上，可使目标 模型很好地保持其原有的个性特征. 另外，将细节 特征提取以后, 利用源基本表情序列和目标基本 表情序列进行联合训练，能够更好地将带有同一 表情的面部模型映射到相同的低维表示, 避免了 因局部细节的尖锐变化导致在求解最小化误差函 数时易出现局部最优的问题, 使联合训练阶段得 到的映射重建函数更加准确，可大大提高算法的 精度.

\section{2 基于拉普拉斯算子的特征提取}

面部模型的基本表情变化往往体现为网格模 型上面积较大的相对平滑区域的变形，而细节信 息位于网格模型上较为尖锐的局部区域. 依据这 种特性，本文对源/目标模型序列进行光顺，将光 顺掉的较为尖锐的局部表情细节称为细节特征信 息, 并用向量来表示这些细节特征信息, 记为细节 特征向量; 光顺后的模型包含基本表情信息, 记为 基本表情模型.

为了提取模型的局部细节，本文利用拉普拉 斯算子 ${ }^{[18]}$ 对模型进行光顺. 对于网格上的顶点, 拉普拉斯算子定义为顶点与其邻接顶点加权组合 的差，它能够表征模型的局部细节. 拉普拉斯光顺 的基本原理是，对每个顶点应用拉普拉斯算子，将 网格上的噪声扩散到顶点的相应邻域中，使模型 表面变得平滑. 该操作能够将局部细节与基本表 情较好分离, 且拉普拉斯光顺简单有效, 时间复杂 度低，可保证本文方法的时间性能.

细节特征提取的步骤: 设网格模型为 $\Psi=(V, E, F)$, 其中 $V$ 为顶点集; $E$ 为边集； $F$ 为 三角面片集; $\left\{\boldsymbol{v}_{1}, \boldsymbol{v}_{2}, \cdots, \boldsymbol{v}_{n}\right\}$ 为点集 $V$ 中的顶点; $n$ 为顶点数. 网格模型的顶点 $\boldsymbol{v}_{i}(i=1,2, \cdots, n)$, 经过 拉普拉斯光顺 ${ }^{[18]}$ 后的新坐标为

$$
\boldsymbol{v}_{i}^{\prime}=\boldsymbol{v}_{i}+\lambda \sum_{j \in N\left(\boldsymbol{v}_{i}\right)} \omega_{i j}\left(\boldsymbol{v}_{j}-\boldsymbol{v}_{i}\right)
$$

其中, $\omega_{i j}=\frac{1}{\left|N\left(\boldsymbol{v}_{i}\right)\right|} ; \sum_{j \in N\left(\boldsymbol{v}_{i}\right)} \omega_{i j}=1 ; N\left(\boldsymbol{v}_{i}\right)$ 表示顶点 $\boldsymbol{v}_{i}$ 的邻接点集合; $\omega_{i j}$ 表示顶点 $\boldsymbol{v}_{i}$ 与邻接点 $\boldsymbol{v}_{j}$ 所构 成的边的权值; $\lambda$ 为控制光顺速度的权值, 取值范 围为 $0 \sim 1$.

利用式(1)对网格模型 $\Psi$ 的顶点进行移动, 得 到光顺后的网格模型 $y=\left(V^{\prime}, E, F\right), y$ 保留了 $\Psi$ 的 基本表情信息, 记为基本表情模型; $v_{i}$ 的位移向量 $\Delta\left(v_{i}\right)$ 记录了 $\Psi$ 上的局部细节信息, 记为细节特征 向量 $\boldsymbol{v}_{i}^{\prime} \boldsymbol{v}_{i}$, 如

$$
\boldsymbol{v}_{i}^{\prime} \boldsymbol{v}_{i}=\Delta\left(\boldsymbol{v}_{i}\right)=\boldsymbol{v}_{i}-\boldsymbol{v}_{i}^{\prime}, \quad i=1,2, \cdots, n
$$

图 2 所示为对网格模型应用拉普拉斯光顺的 效果. 可见, 经过光顺后, 模型的个性细节特征信 息可以被很好地提取并保存，方便用于后续的细 节特征恢复.

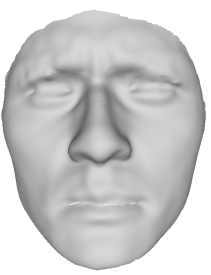

a. 待光顺模型

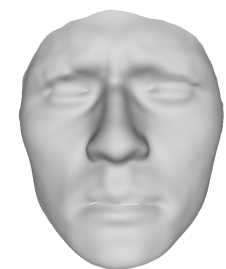

b. 光顺后基本表情模型
图 2 表情细节特征提取前后效果图

对源/目标模型序列中的每一个网格模型利用 式(1)(2)进行细节特征提取, 即可得到源/目标基本 表情模型序列、源基本表情模型和目标模型细节特 征向量.

\section{3 基本表情迁移}

为实现源模型基本表情向目标模型的迁移, 本文采用联合训练的思想，将提取细节特征后的 源/目标基本表情模型映射到低维表示; 然后根据 低维表示重建目标模型, 且重建的目标模型与源 模型具有相同或相近的基本表情. 本文对非线性 联合学习方法 ${ }^{[19]}$ 进行了改进，将其中使用的无监 督回归(dimensionality reduction by unsupervised regression，nDRUR)方法 ${ }^{[20]}$ 置换为 pDRUR 方法, 并利用梯度下降法进行迭代优化, 目的是降低联 合训练的时间与空间消耗. 本文方法保留了表情 投影约束的思想, 使得其能够将表情一致的源/目 标模型序列映射到相同的低维表示.

基本表情迁移分为 2 步. 
Step1. 联合训练. 从源/目标基本表情模型序列中 分别提取 $m$ 个表情模型, 要求提取出的 2 组样本表情一 一对应, 分别表示为样本集合 $Y_{1}=\left[y_{11}, y_{12}, \cdots, y_{1 m}\right]$ 和 $Y_{2}=\left[y_{21}, y_{22}, \cdots, y_{2 m}\right]$. 其中, $y_{i j}$ 表示一个样本表情模型, $y_{1 j}$ 和 $y_{2 j}$ 具有相同表情. 利用联合训练对 2 组样本表情 进行函数求解, 得到映射函数和重建函数.

Step2. 源表情传递. 输人源基本表情模型序列中 任意一个未参与训练的面部模型 $y_{1}^{\prime}$, 利用函数重建出 与 $y_{1}^{\prime}$ 具有相同或相近表情的目标模型 $y_{2}^{\prime}$.

\section{1 联合训练}

联合训练得到对应于源/目标基本表情模型样 本集合 $Y_{1}$ 和 $Y_{2}$ 的 2 组映射/重建函数 $\left\{F_{i} / f_{i}\right\}$, 其 中，映射函数能将源/目标基本表情模型样本集合 中具有相同表情的面部模型映射到相同的低维表 示 $X=\left[x_{1}, \cdots, x_{m}\right], x_{j}$ 为 $y_{1 j}$ 和 $y_{2 j}$ 相同的低维表示. 重建函数能够根据低维表示重建出源/目标基本表 情模型. 本文方法引人投影约束的思想来满足上 述要求，该约束将源基本表情样本集合 $Y_{1}$ 和目标 基本表情样本集合 $Y_{2}$ 中表情一致的面部模型投影 到同一低维表示. 约束方程 ${ }^{[19]}$ 为 $\forall_{i} F_{i}\left(y_{i j}\right)=x_{j}$; 映 射/重建函数求解公式 ${ }^{[19]}$ 为

$$
\begin{aligned}
& \arg \min _{X,\left\{f_{i}\right\},\left\{F_{i}\right\}} \sum_{i=1}^{2} E_{i}\left(X, f_{i}, F_{i}\right)= \\
& \arg \min _{X,\left\{f_{i}\right\},\left\{F_{i}\right\}} \sum_{i=1}^{2}\left\|E_{f_{i}}\left(X, f_{i}\right)+E_{F_{i}}\left(X, F_{i}\right)\right\|
\end{aligned}
$$

其中,

$$
\begin{aligned}
& E_{f_{i}}\left(X, f_{i}\right)=\sum_{j=1}^{m}\left\|y_{i j}-f_{i}\left(x_{j}\right)\right\|^{2}+\lambda_{f_{i}} R_{f_{i}}\left(f_{i}\right) ; \\
& E_{F_{i}}\left(X, F_{i}\right)=\sum_{j=1}^{m}\left\|x_{j}-F_{i}\left(y_{i j}\right)\right\|^{2}+\lambda_{F_{i}} R_{F_{i}}\left(F_{i}\right) .
\end{aligned}
$$

式(3)表示源基本表情模型样本集合和目标基本表 情模型样本集合上误差函数的和. 其中, $E_{i}\left(X, f_{i}, F_{i}\right),(i=1,2)$ 表示第 $i$ 个样本集合的误差 函数; $\lambda_{f_{i}} R_{f_{i}}\left(f_{i}\right)$ 和 $\lambda_{F_{i}} R_{F_{i}}\left(F_{i}\right)$ 是为防止奇异解的产 生而引人的正则项， $\lambda$ 表示权值， $\|\cdot\|$ 表示 2-范数. 本文利用改进的 pDRUR 方法求解上述问题. 首先 使用拉普拉斯特征映射得到源基本表情模型样本 集合 $Y_{1}$ 和目标基本表情模型样本集合 $Y_{2}$ 在低维空 间中的初始坐标 $C_{1}$ 和 $C_{2}$, 拉普拉斯特征映射能够 将原空间中相互靠近的点在降维后依然尽可能地 靠近，从而保持原有的局部结构. 此外，由于样本 集合 $Y_{1}$ 和样本集合 $Y_{2}$ 中的模型表情一一对应，经 过拉普拉斯特征映射后的 $C_{1}$ 和 $C_{2}$ 理论上基本接
近, 因此本文设 $X$ 为 $C_{1}$ 和 $C_{2}$ 的均值, 即 $X=\theta_{1} C_{1}+$ $\theta_{2} C_{2}, \theta_{1}=\theta_{2}=\frac{1}{2}$. 本文将在第 5.3 节通过实验分析 不同初值对联合训练误差的影响, 进一步验证 $X$ 选择该初始值的合理性. 初值 $X$ 一旦确定, 算法 便可以迭代优化自适应过程和映射过程求解目标 参数.

pDRUR 方法是对 nDRUR 方法的改进, 它们 的求解过程相似, 都是交替优化映射过程和自适 应过程，但 pDRUR 的时间复杂度是线性的，使得 训练过程的推导速度更快. 这 2 种方法虽然具有相 同的 $(X, f, F)$ 的目标求解函数, 但 pDRUR 求解的 $f$ 和 $F$ 是特殊的参数化形式, 并且正则项 $R_{f}(f)$ 和 $R_{F}(F)$ 是对 $f$ 和 $F$ 参数的约束. pDRUR 针对单 组样本进行训练, 映射过程采用高斯牛顿法. 高斯 牛顿法在寻找目标函数最小值的过程中仅需迭代 计算 1 对参数即可, 而本文需要求解联合目标函 数, 要求算法能提供 2 对包含各自样本集合特性的 参数, 高斯牛顿法无法合理地整合 2 对参数, 因而 无法求解双样本集合的共同低维表示 $X$. 因此, 为求解联合目标函数, 本文将高斯牛顿法替换为 梯度下降法, 并对联合目标函数中 $X$ 的梯度进行 重新定义，提出新的 pDRUR.

Step1. 自适应过程. 自适应过程是对式(3)中 2 个 误差函数 $E_{f_{i}}\left(X, f_{i}\right)$ 和 $E_{F_{i}}\left(X, F_{i}\right)$ 的独立最小化, 是 2 个 标准的回归方程, 回归方程由 $D$ 个高斯基函数的和与 偏置项构成. 以 $f$ 为例, 其回归方程 ${ }^{[17]}$ 表示为

$$
f(x)=\boldsymbol{W} \Phi(x)+w=\sum_{d=1}^{D} w_{d} \varphi_{d}(x)+w .
$$

其中, $\Phi(x)$ 表示 $D$ 个高斯基函数的和; $\varphi_{d}(x)=$ $\exp \left(-\frac{1}{2}\left\|\left(x-\mu_{d}\right) / \sigma\right\|^{2}\right)$ 表示基函数; $\mu_{d}$ 表示基函数的 中心，通过 $k$ 均值聚类算法求解； $\sigma$ 表示基函数的宽度， 通过网格搜索求解; $w$ 表示偏置项; $\boldsymbol{W}$ 表示权重, 求解 方程 ${ }^{[17]}$ 为

$$
\begin{gathered}
\min _{\boldsymbol{W}}\left\|Y-\boldsymbol{W} \boldsymbol{G}_{x y}-w \boldsymbol{l}^{\mathrm{T}}\right\|^{2}+\lambda \operatorname{tr}\left(\boldsymbol{W} \boldsymbol{G}_{x x} \boldsymbol{W}^{\mathrm{T}}\right) \Rightarrow \\
\boldsymbol{W}\left(\boldsymbol{G}_{x y} \boldsymbol{G}_{x y}^{\mathrm{T}}+\lambda \boldsymbol{G}_{x x}\right)=\left(Y-w \boldsymbol{l}^{\mathrm{T}}\right) \boldsymbol{G}_{x y}^{\mathrm{T}} .
\end{gathered}
$$

其中, $\boldsymbol{l}$ 表示 $m$ 个 1 组成的单位列向量; $\boldsymbol{G}_{x y}$ 表示由 $\varphi_{d}\left(x_{j}\right)$ 构成的矩阵; $\boldsymbol{G}_{x x}$ 表示由 $\varphi_{d}\left(\mu_{d}\right)$ 构成的矩阵; $\lambda \operatorname{tr}\left(\boldsymbol{W} \boldsymbol{G}_{x x} \boldsymbol{W}^{\mathrm{T}}\right)$ 表示正则项; $\lambda$ 为用户自定义参数, 取值 大于或等于 0 . 将 $w=\frac{1}{m}\left(Y-\boldsymbol{W} \boldsymbol{G}_{x y}\right) \boldsymbol{l}$ 代人到 $\boldsymbol{W}$ 的求解方 程中, 得到

$$
\boldsymbol{W}\left(\boldsymbol{G}_{x y} \boldsymbol{G}_{x y}^{\mathrm{T}}+\lambda \boldsymbol{G}_{x x}-\frac{1}{m}\left(\boldsymbol{G}_{x y} \boldsymbol{l}\right)\left(\boldsymbol{G}_{x y} \boldsymbol{l}\right)^{\mathrm{T}}\right)=Y\left(\boldsymbol{I}-\frac{1}{m} \boldsymbol{l}^{\mathrm{T}}\right) \boldsymbol{G}_{x y}^{\mathrm{T}} ;
$$


$\boldsymbol{W}$ 由正定线性系统求解. 对于 $F$ 而言, 由于 $Y$ 不随迭 代过程发生变化，因此算法能够快速地计算出基函数的 中心和宽度; 但对于 $f$ 而言, 由于 $X$ 不是恒定的, 算法 需要不断地进行迭代更新. 在每次迭代或每几次迭代的 过程中，本文采用 $k$ 均值算法重新计算基函数的中心， 并以上一次迭代的值进行初始化.

Step2. 映射过程. 固定参数化的 $f_{i}$ 和 $F_{i}, i=1,2$; 采用梯度下降法对 $X$ 进行迭代优化. 从数学的角度分 析，梯度的方向是函数变化速度最快的方向，梯度下降 法的核心思想就是通过迭代的方式寻找目标函数的最 小值. 梯度下降法操作简便, 且求解出的共同低维表示 $X$ 可以包含各样本集合的特性，在迭代过程中只需要 不断地计算梯度, 便可以实现参数的更新. 本文 $X$ 的梯 度为式(3)中 2 组误差函数的梯度之和, 即 $\sum_{i=1}^{2} \frac{\partial E_{i}}{\partial X}$.

\section{2 源表情传递}

本文利用联合训练求解的 2 组映射/重建函数 $\left\{F_{i} / f_{i}\right\}, i=1,2$, 将源基本表情模型 $y_{1}^{\prime}$ 的基本表情 传递给目标模型 $y_{2}^{\prime}$. 具体地, 首先利用源基本表 情模型序列的映射函数 $F_{1}$ 将 $y_{1}^{\prime}$ 的基本表情映射到 低维表示 $x^{\prime}$; 然后利用目标基本表情模型序列的 重建函数 $f_{2}$ 对 $x^{\prime}$ 重建, 得到与 $y_{1}^{\prime}$ 具有相同或相近 表情的目标模型 $y_{2}^{\prime}$ ，即 $x^{\prime}=F_{1}\left(y_{1}^{\prime}\right), y_{2}^{\prime}=f_{2}\left(x^{\prime}\right)$.

图 3 所示为经过源表情传递后重建出的目标 模型效果. 可见，目标模型已经具有了与源模型相 同或非常相近的基本表情.

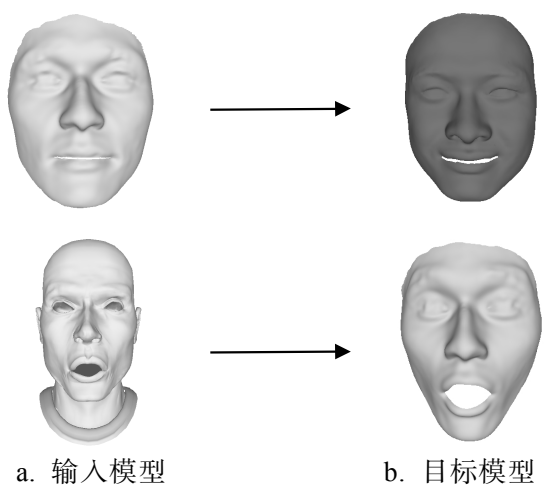

图 3 源表情传递效果

\section{4 目标模型细节特征恢复}

基本表情迁移通过函数重建的方式仅将源模 型的基本表情传递给目标模型，下面需要对目标 模型原有的细节特征进行恢复，以重建其精细的 个性化细节信息。本文在细节特征提取阶段已将 目标模型的细节特征保存为向量的形式，但目标 模型的网格形状在经过表情传递后发生了较大变
化, 将这些细节特征向量直接叠加到目标模型上 对应的顶点处是不合理的. 因此, 本文提出依据目 标模型基本表情变化进行细节特征向量调整的新 策略. 通过对细节特征向量的方向和大小进行调 整, 再叠加回目标模型的方式, 既很好地恢复了目 标模型的个性化细节信息, 又使得这些细节与新 表情相符，保证了表情的真实自然.

\section{1 细节特征向量方向调整}

由于细节特征信息多存在于局部微小区域, 且不会发生大幅度变动, 因此本文依据提取细节 特征后的目标模型在进行源基本表情传递前后各 个顶点的法向变化情况，将它们的细节特征向量 方向调整一定角度，使其适应新表情的变化. 目标 模型上各个顶点的法向量由其邻域三角面片的法 向量加权平均得到，它可以反映顶点局部区域的 几何特征. 由于法向量与局部区域切平面垂直，因 此其法向可反映局部区域的朝向. 表情变化前后, 因细节区域变化较小，每个顶点处的细节特征向 量相对于该顶点局部区域的朝向应该基本保持不 变，即与该顶点的法向的夹角保持不变. 基于此, 本文对源基本表情传递后的目标模型上每个顶点 处的细节特征向量的方向进行调整，使得调整后 的细节特征向量与顶点处的法向量夹角, 与源表 情传递前目标模型上该顶点处的细节特征向量与 顶点法向量的夹角相等.

本文方法可将任意源模型的表情传递到任意 目标模型上，为叙述方便，取目标模型序列中的任 意表情模型作为参考模型, 即原始目标模型. 如图 4 所示, 将原始目标模型上的某一顶点记为 $\boldsymbol{v}_{i}$, $i=1,2, \cdots, n$, 利用式(1), 将顶点 $\boldsymbol{v}_{i}$ 经过细节特征提 取后的新位置记为光顺点 $v_{i}^{\prime}$; 利用式(2), 将顶点 $\boldsymbol{v}_{i}$ 经过细节特征提取后的位移量记为细节特征向 量 $\boldsymbol{v}_{i}^{\prime} \boldsymbol{v}_{i}=\boldsymbol{v}_{i}-\boldsymbol{v}_{i}^{\prime}$. 经源表情传递后, 将光顺点 $\boldsymbol{v}_{i}^{\prime}$ 移动 到的新位置记为初步表情点 $\boldsymbol{q}_{i}$; 将光顺点 $\boldsymbol{v}_{i}^{\prime}$ 与初 步表情点 $q_{i}$ 的位移量记为向量 $\boldsymbol{v}_{i}^{\prime} \boldsymbol{q}_{i}=\boldsymbol{q}_{i}-\boldsymbol{v}_{i}^{\prime}$; 将光 顺点 $\boldsymbol{v}_{i}^{\prime}$ 处的法向量记为 $\boldsymbol{N}_{v_{i}^{\prime}}$; 将初步表情点 $\boldsymbol{q}_{i}$ 的 法向量记为 $\boldsymbol{N}_{\boldsymbol{q}_{i}}$; 将法向量 $\boldsymbol{N}_{\boldsymbol{v}_{i}^{\prime}}$ 与细节特征向量 $\boldsymbol{v}_{i}^{\prime} \boldsymbol{v}_{i}$ 的夹角记为 $\alpha_{i}$; 将法向量 $\boldsymbol{N}_{q_{i}}$ 与 $\boldsymbol{q}_{i}$ 点待求的新 细节特征向量 $\boldsymbol{q}_{i} \boldsymbol{p}_{i}$ 的夹角记为 $\beta_{i}$. 令 $\alpha_{i}=\beta_{i}$, 可得 $\cos \alpha_{i}=\cos \beta_{i}$, 即满足

$$
\frac{\boldsymbol{N}_{v_{i}^{\prime}} \times \boldsymbol{v}_{i}^{\prime} \boldsymbol{v}_{i}}{\left(\left|\boldsymbol{N}_{v_{i}^{\prime}}\right| \times\left|\boldsymbol{v}_{i}^{\prime} \boldsymbol{v}_{i}\right|\right)}=\frac{\boldsymbol{N}_{q_{i}} \times \boldsymbol{q}_{i} \boldsymbol{p}_{i}}{\left(\left|\boldsymbol{N}_{q_{i}}\right| \times\left|\boldsymbol{q}_{i} \boldsymbol{p}_{i}\right|\right)}
$$

式(4)存在多个解, 本文方法将待求的新细节特征 
向量 $\boldsymbol{q}_{i} \boldsymbol{p}_{i}$ 限定在法向量 $\boldsymbol{N}_{q_{i}}$ 和向量 $\boldsymbol{q}_{i} \boldsymbol{q}_{i}^{\prime}\left(\boldsymbol{q}_{i} \boldsymbol{q}_{i}^{\prime}\right.$ 与 $\boldsymbol{v}_{i}^{\prime} \boldsymbol{v}_{i}$ 平行)所构成的平面内, 如图 4 所示. 由此通过求解 式(4)可得到唯一的新细节特征向量 $\boldsymbol{q}_{i} \boldsymbol{p}_{i}$ 的新方向.

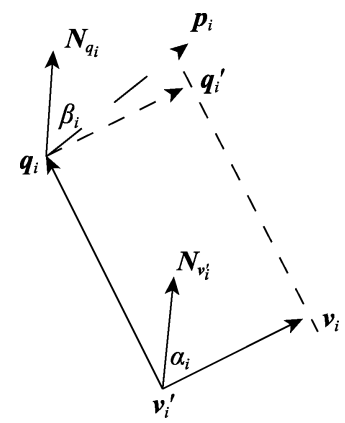

图 4 细节特征向量方向调整示意图

\section{2 细节特征向量大小调整}

经源表情传递后的目标模型的几何形状发生 了较大变化, 这些几何形状的变化也会对局部细 节信息产生影响，因此直接将原始大小的特征向 量叠加到目标模型上将无法准确地反映目标模型 的细节信息. 另外，由于细节信息往往是局部的微 小差异，因此本文方法不能仅对细节特征向量进 行简单地全局缩放，而需要通过局部顶点的比例 因子对每个特征向量的大小进行单独调整. 借用 Noh 等 ${ }^{[21]}$ 的思想, 将局部顶点的特征向量大小的 比例因子定义为该顶点在源表情传递前后，其邻 域三角形面积和的比值.

本文中具有新表情的目标模型实际是通过函 数重建的方式得到, 详见第 4.1 节. 本文方法将细 节特征提取后的任意一个目标表情模型作为原始 目标模型, 即作为具有新表情目标模型的参考模 型. 将 $\boldsymbol{q}_{i}$ 经过细节特征向量方向调整后的坐标记 为 $\boldsymbol{p}_{i}$, 顶点 $\boldsymbol{q}_{i}$ 处的特征向量大小的比例因子 $r_{i}$ 定 义为 $r_{i}=S\left(\boldsymbol{q}_{i}\right) / S\left(\boldsymbol{v}_{i}^{\prime}\right)$. 其中, $S\left(\boldsymbol{q}_{i}\right)$ 是源表情传递后 目标模型顶点 $\boldsymbol{q}_{i}$ 的邻域三角形的面积和; $S\left(v_{i}^{\prime}\right)$ 是 源表情传递前顶点邻域三角形的面积和, 即细节 特征提取后的原始目标模型的顶点 $v_{i}^{\prime}$ 的邻域三角 形的面积和.

本文以细节特征向量 $\boldsymbol{v}_{i}^{\prime} \boldsymbol{v}_{i}$ 的模长 $\left|\boldsymbol{v}_{i}^{\prime} \boldsymbol{v}_{i}\right|$ 来表征 大小, 新向量大小的调整公式为 $\left|\boldsymbol{v}_{i}^{\prime} \boldsymbol{v}_{i}\right|^{\prime}=r_{i} \times\left|\boldsymbol{v}_{i}^{\prime} \boldsymbol{v}_{i}\right|$.

获得新模长后，本文将在局部坐标系中按照 向量 $\boldsymbol{q}_{i} \boldsymbol{p}_{i}$ 方向对模长 $\left|\boldsymbol{v}_{i}^{\prime} \boldsymbol{v}_{i}\right|^{\prime}$ 进行坐标轴分解，从而 计算出新坐标 $\boldsymbol{p}_{i}^{\prime}$.

图 5 所示为对源表情传递后的目标模型进行 细节特征恢复的效果. 可见, 恢复后的面部模型眉 毛区域的皱纹明显增多，个性细节特征更加突出.

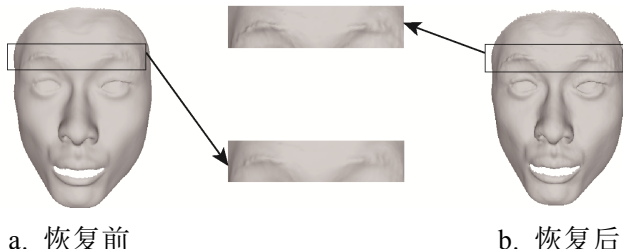

图 5 细节特征恢复前后模型效果图

为进一步验证细节特征恢复方法的有效性, 本文取同一个面部表情序列，将其中的不同帧分 别作为源模型和目标模型, 使得经过表情传递并 细节恢复后的目标模型有明确的源参考模型, 便 于直观比较和定量分析效果. 具体地, 先对同一人 脸模型序列进行细节特征提取、pDRUR 训练得到 该序列的映射重建函数; 然后输人序列中任意一 个未参与训练的模型作为源参考模型，经过映射、 重建、细节恢复后，观察并比较得到的新表情模型 与源参考模型的视觉差异和空间误差.

表 1 所示为细节特征的恢复效果及误差分析. 通过比较细节特征恢复后的模型和源参考模型发 现，两者的细节区域高度一致; 另外，两者之间的 误差非常小. 可见，细节特征恢复方法是有效的， 其很好地恢复了目标模型上的表情细节信息，且 这些表情细节与新表情相符，没有违和感.

表 1 细节特征恢复效果及误差分析

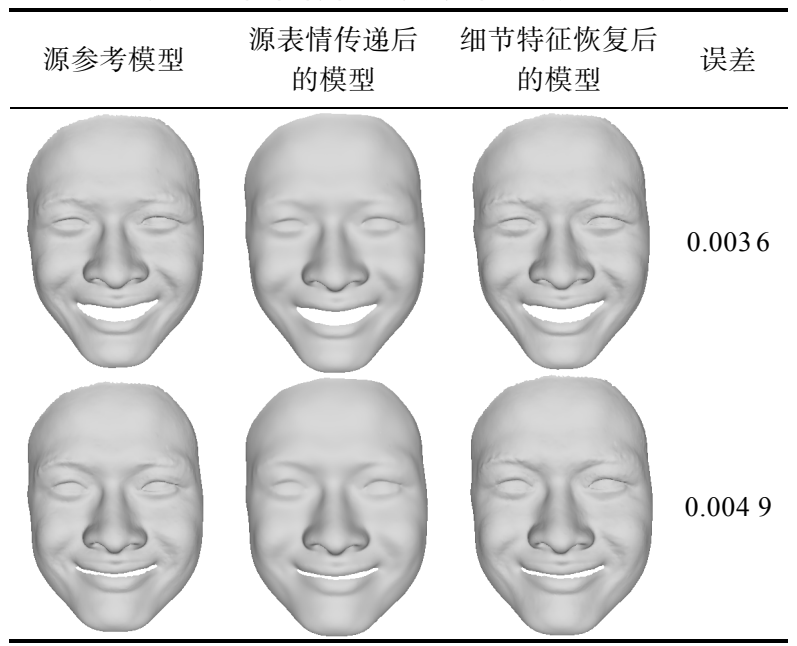

注. 误差为细节特征恢复后的模型与源参考模型间所有 对应顶点的空间欧几里得度量平方和的均值.

\section{5 实验及结果分析}

本节在证明了本文方法细节提取的有效性， 并展示了其完整实验结果后，比较了不同初值选 择对联合训练结果的影响, 最后列出了本文方法 的时间性能. 本文方法由 Matlab 和 Python 语言实 
现, 运行环境为 Windows 10 系统, 计算机配置为 $2.60 \mathrm{GHz}$ Intel Core i5-3230M CPU, 8 GB 内存.

\section{1 细节提取的有效性}

第 2.1 节中提到，细节特征提取后的基本表情 模型能够更好地进行函数拟合，提高映射/重建函 数的精度, 从而准确地将源模型的基本表情传递 给目标模型. 为进一步证明方法的有效性, 本文从 理论和实验 2 个方面进行分析. 通过理论分析发 现，联合训练中的投影约束部分并不会因为模型 是否光顺受到影响, 受影响的部分是联合训练中 最为核心的 pDRUR 部分，而 pDRUR 本身具有将 同种属性模型映射到同一低维表达的能力, 即具

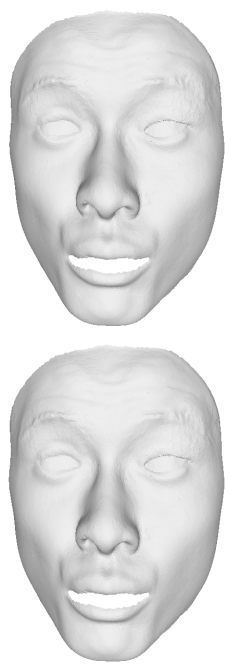

a. 输人模型

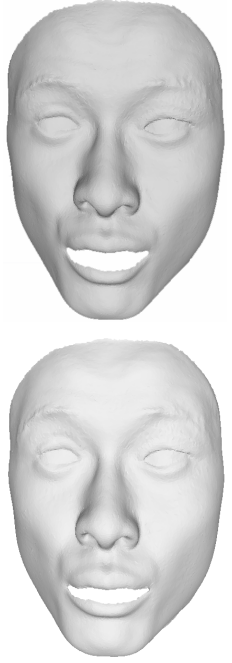

b. 重建结果

有获得映射/重建函数的能力. 为直观地呈现利用 细节提取后的模型以及未提取过细节的模型分别 进行表情传递后的比较结果，本文设计在同一个 面部模型之间进行不同表情传递的实验，以便使 传递后的目标模型的新表情具有明确的参考模型. 具体地，对同一个人的面部模型序列，利用对细节 提取前后的模型分别进行 pDRUR 训练, 得到此模 型序列的映射/重建函数; 然后从序列中任取一个 未参与训练的模型作为输人模型, 经过映射/重建 后, 比较 2 种方式获得的模型与输人模型的一致程 度. 图 6 所示为对细节提取前后模型应用 pDRUR 的实验对比.

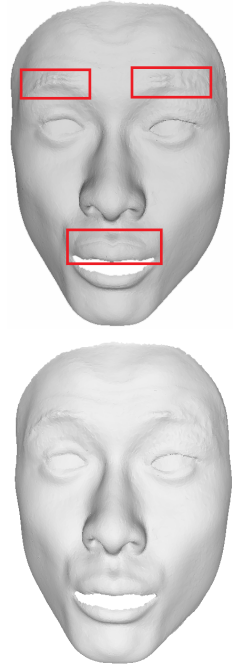

c. $\mathrm{a}$ 与 $\mathrm{b}$ 的叠加对照

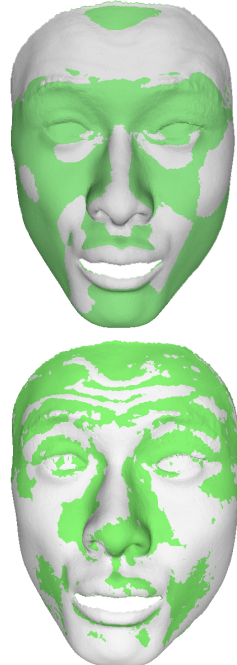

d. a 与 $b$ 带颜色的叠加对照

图 6 对未提取以及提取过细节的模型分别应用 pDRUR 的效果对比

在图 6 中，第 1 行是利用未提取细节的模型获 得的结果，第 2 行是利用提取细节后的模型获得的 结果. 从图 $6 \mathrm{~b}$ 可以看出, 利用未提取细节的模型 获得的重建模型嘴部张开幅度变大，利用提取细 节后的模型获得的重建模型嘴部张开幅度与输人 模型一致. 从图 6c 可以看出, 利用未提取细节的 模型获得的重建模型不仅嘴部拟合不准确，且眉 毛部分与输人模型差异很大; 而利用提取细节后 的模型获得的重建模型，可以很好地逼近输人模 型, 未出现高度与幅度的明显差异. 从图 $6 \mathrm{~d}$ 可以 看出，利用未提取细节的模型获得的重建模型即 使在平坦区域也出现大面积拟合不准确的情况; 而利用提取细节后的模型获得的重建模型出现差 异的部分基本位于细节区域，本文方法还会对这 些细节信息进行单独恢复. 可见, 预先光顺掉细节 特征信息对提高函数拟合的准确度是非常有效的.

\section{2 表情迁移}

本节从视觉对比和定量分析 2 个方面比较非 线性联合学习方法 ${ }^{[19]}$ 与本文方法获得的表情迁移 结果. 由于迁移到目标模型上的新表情, 是目标模 型之前不存在的表情, 为解决迁移后目标模型缺 少参考模型而难以进行定量分析的问题, 本文设 计在同一个面部模型序列之间进行表情迁移的实 验，以保证每个新表情都有对应的参考模型. 具体 地, 对同一个人的面部模型序列, 分别应用非线性 联合学习方法和本文方法得到此模型序列的映射/ 重建函数; 然后从序列中任取一个未参与训练的 模型作为输人模型, 经过各自方法的映射/重建以 及本文方法的细节特征恢复等操作后, 比较 2 种方 法获得的模型与输人模型的视觉一致程度以及模 型之间的误差值.

表 2 所示为对多组不同角色的同一模型序列 
分别应用非线性联合学习方法与本文方法进行表 情迁移的效果对比. 其中, 误差表示为各自方法得 到的模型与源模型所有对应顶点的空间欧几里得 度量平方和的均值. 通过观察可以发现, 在同一模 型序列之间进行表情传递时，2 种方法获得的表情 效果在视觉上差异不大, 但通过误差比较可以发 现，本文方法的误差均低于非线性联合学习方法 的误差. 在第 4 行模型中, 2 种方法的误差都高于 前 3 行, 主要原因是第 4 行模型的顶点数远高于前 3 行, 顶点数的增加对 2 种方法的联合训练阶段都 产生了一定影响.

图 7 图 9 所示为对不同模型之间应用非线性 联合学习方法 ${ }^{[19]}$ 与本文方法进行表情迁移的视觉 效果对比图. 图 7 中的实验数据来自 COMA 数据
集 ${ }^{[22]}$ 中的 2 组头部模型.

首先, 由图 7 前 2 行的实验结果可见, 利用非 线性联合学习方法得到的表情模型, 其细节特征 有明显缺失(如框内所示眉眼处)，且第 2 行中的表 情迁移结果与源表情模型在整体表情幅度上有一 定差异; 而本文方法不仅准确地实现了表情的传 递, 而且很好地保留了目标模型的细节特征. 为证 明本文方法可以在 2 个模型序列之间进行任意的 表情传递，实验将源模型与目标模型进行调换，得 到图 7 后 2 行的表情传递结果. 通过观察可以发现, 联合学习方法获得的表情模型不仅缺少细节信息, 而且存在表情传递失败的情况; 而本文方法在实 现表情正确传递的同时，有效地保留了目标模型 的局部细节.

表 22 种方法对同一模型序列表情迁移效果对比

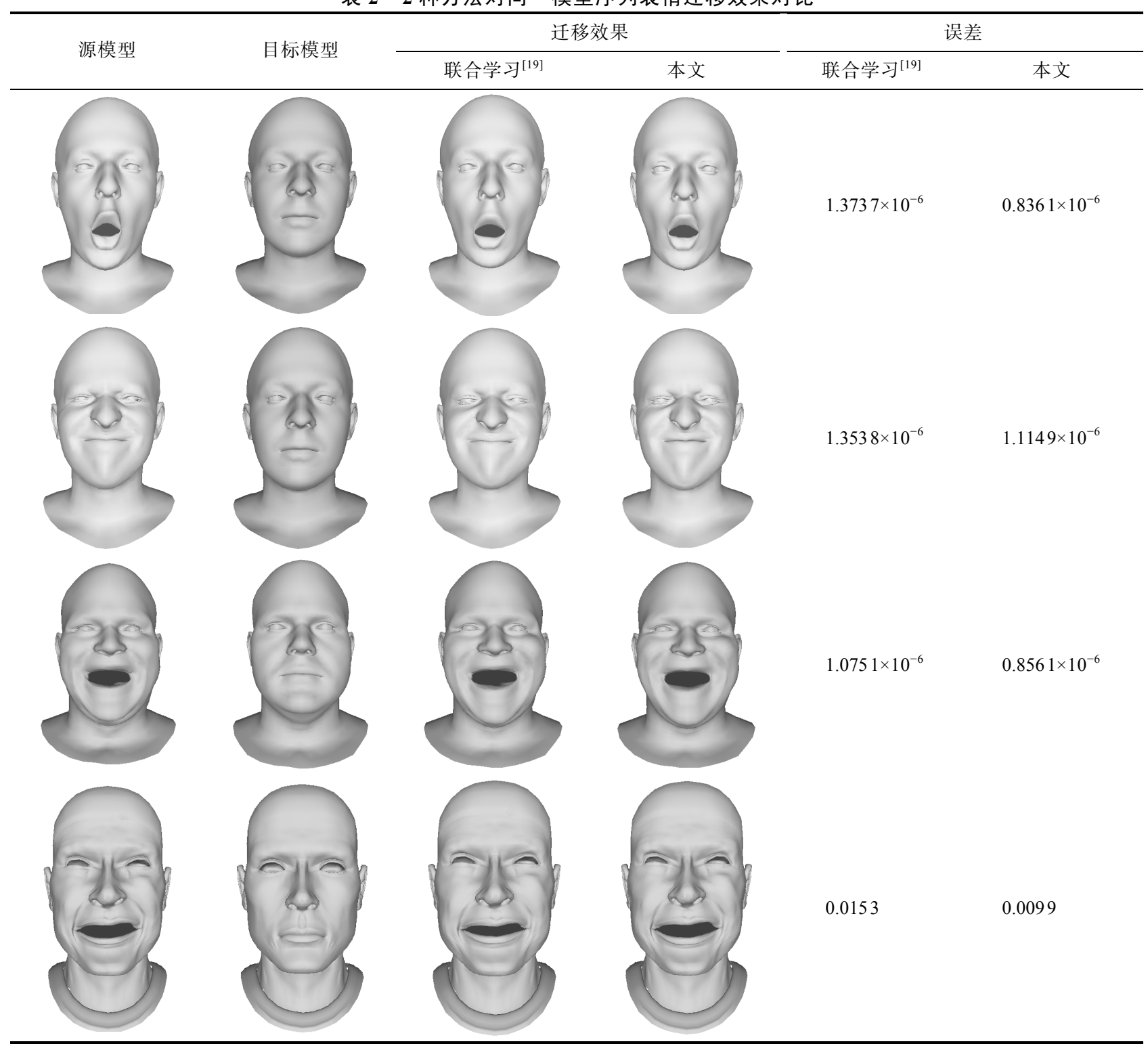




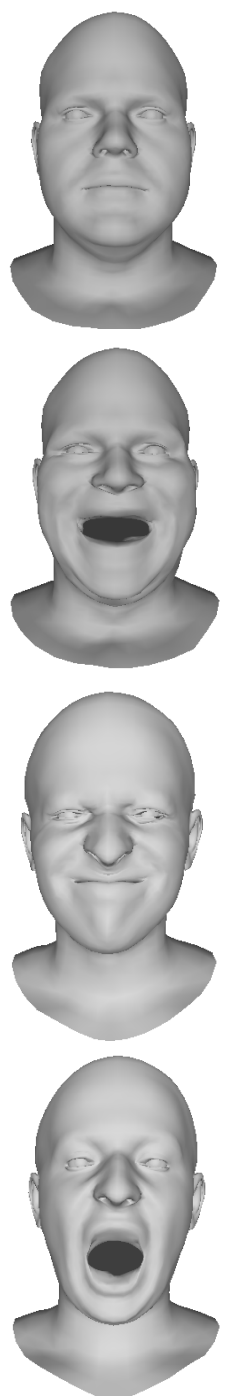

a. 源表情模型
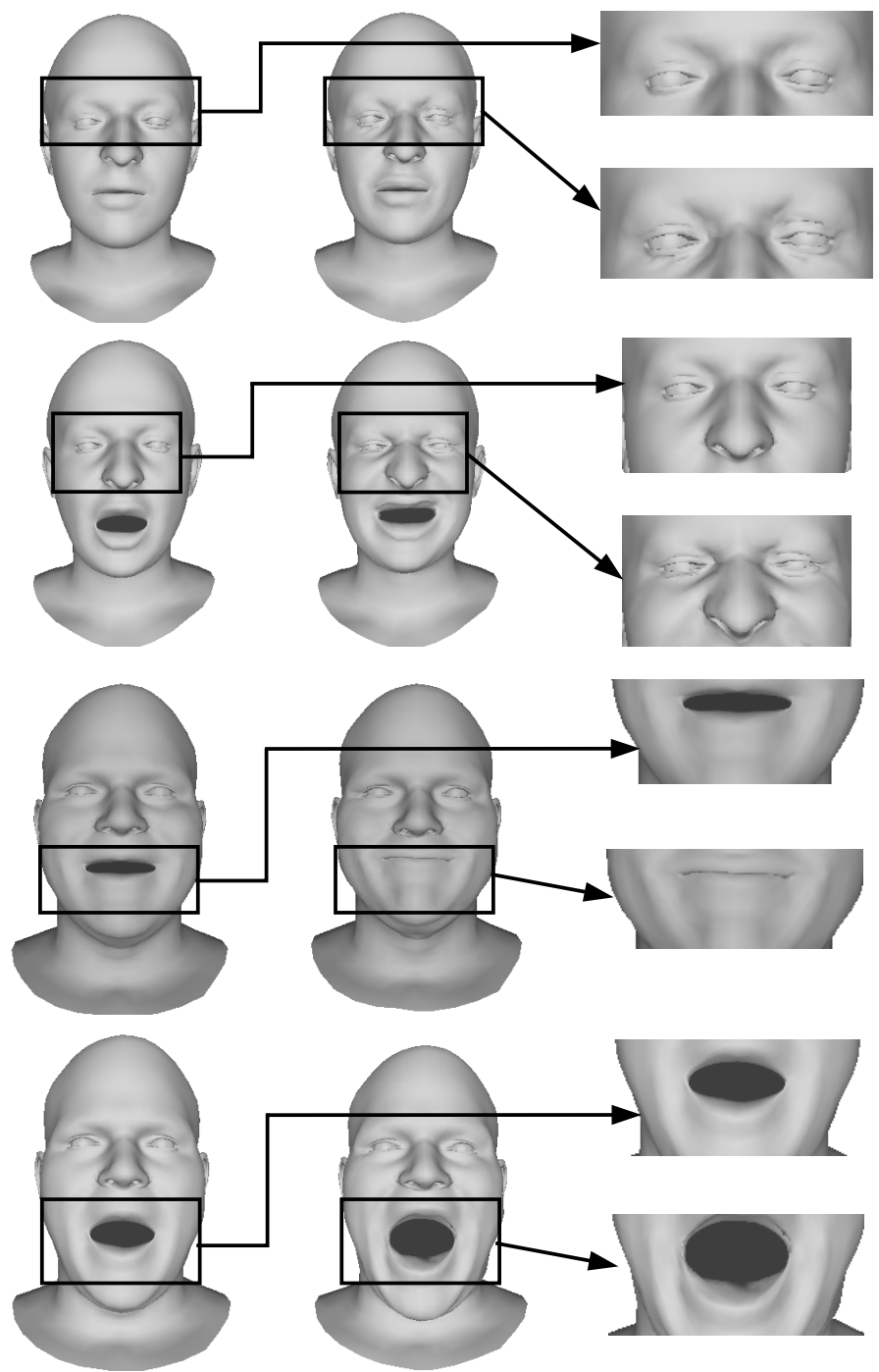

b. 联合学习[19]

c. 本文

d. 细节放大对比

图 7 不同方法表情迁移效果对比 1
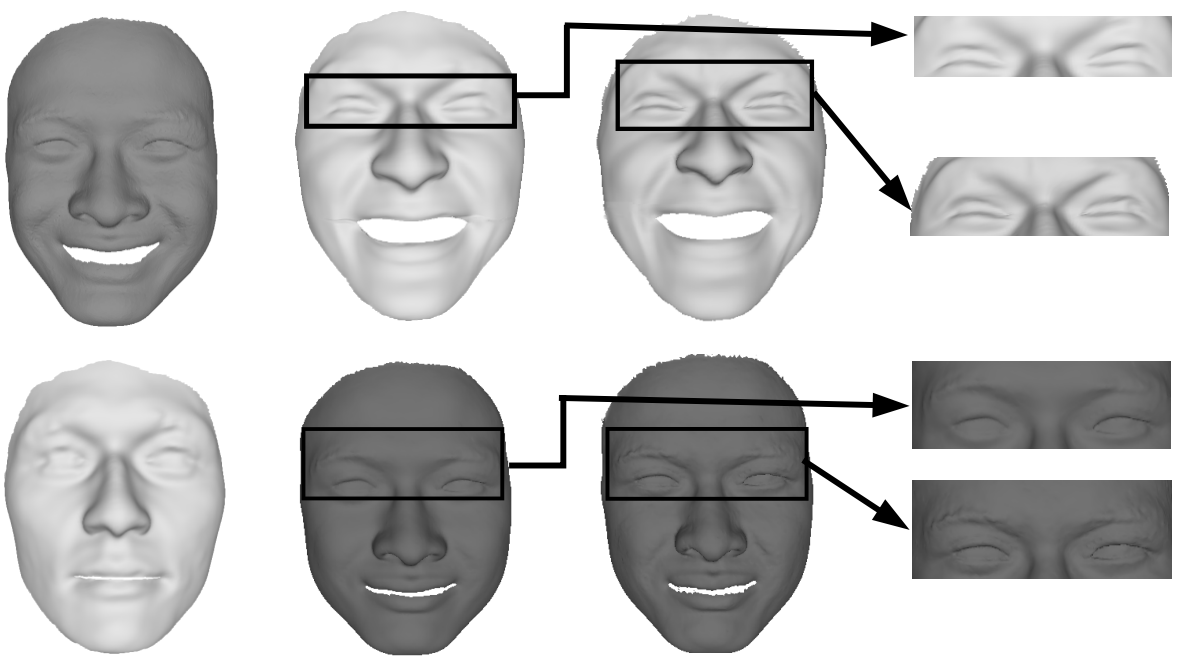

a. 源表情模型

b. 联合学习[19]

c. 本文

d. 细节放大对比

图 8 不同方法表情迁移效果对比 2 

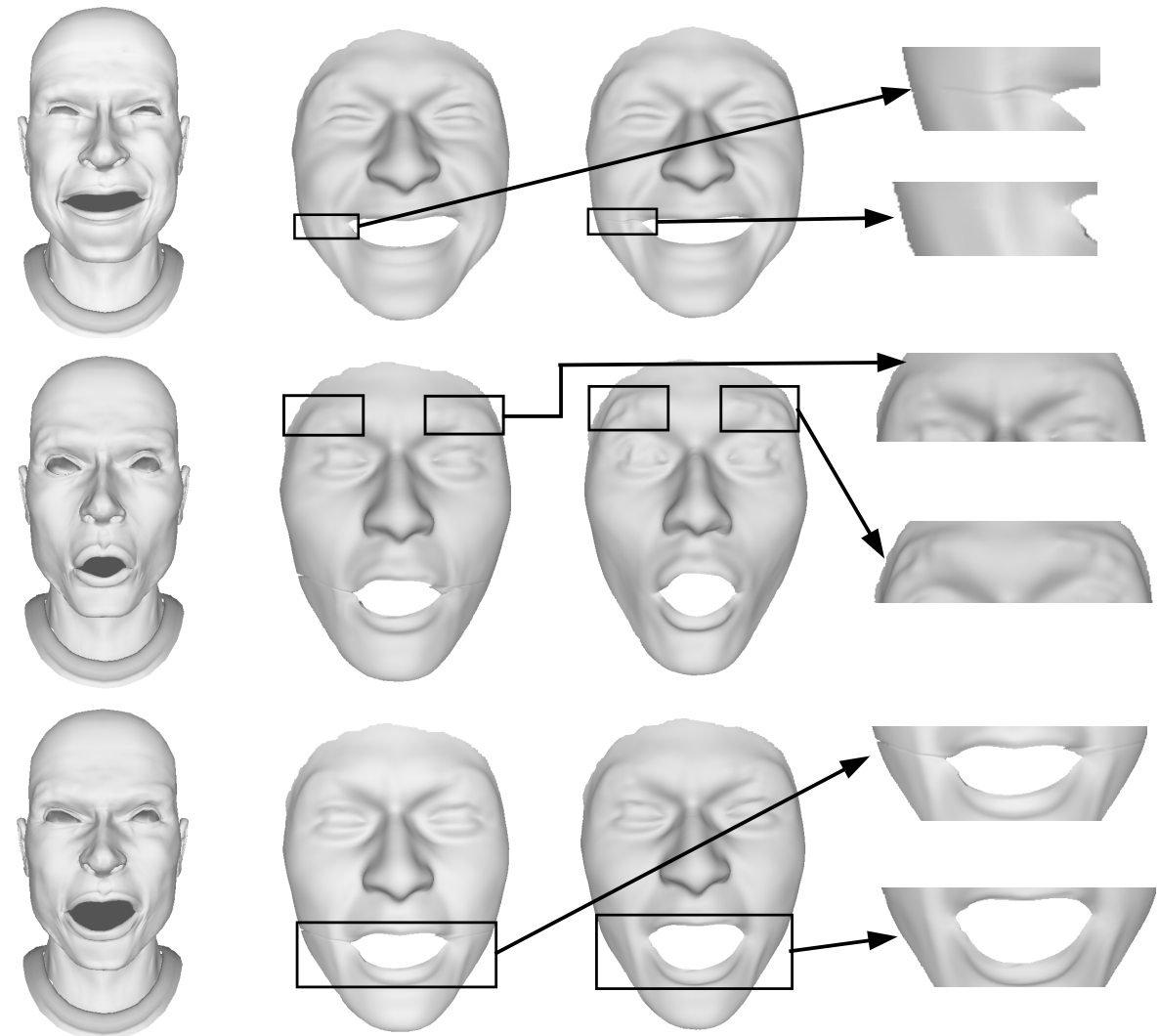

a. 源表情模型

b. 联合学习 ${ }^{[19]}$

c. 本文

d. 细节放大对比

图 9 不同方法表情迁移效果对比 3

对 2 组面部模型, 分别利用非线性联合学习方 法 ${ }^{[19]}$ 与本文方法进行表情迁移, 效果比较如图 8 所示. 由图 8 第 1 行的实验结果可见, 利用联合学 习方法得到的模型不仅嘴部区域出现了裂痕, 且 细节特征不够明显，而本文方法较好地实现了表 情的传递，且恢复了目标模型的细节信息. 第 2 行 是将源模型与目标模型进行调换后得到的表情迁 移结果. 通过比较可见，尽管 2 种方法都实现了表 情的传递，但本文方法得到的模型细节特征更加 明显。

对头部模型组与面部模型组, 分别利用非线 性联合学习方法 ${ }^{[19]}$ 与本文方法进行表情迁移, 效 果比较如图 9 所示. 图 9 说明本文方法不仅能够实 现头部模型与头部模型之间、面部模型和面部模型 之间的表情传递，对于头部模型与面部模型之间 的表情传递同样有效. 通过观察可以发现，联合学 习方法得到的新表情模型的嘴部区域全部出现了 裂痕，且第 2 行模型的眼眶眉毛区域相较于本文方 法得到的结果，明显缺少细节信息，因此整体表情 迁移效果不准确. 本文方法不仅实现了模型之间 基本表情的准确传递，而且完好地保持了目标模 型的细节特征. 针对第 3 行源模型下嘴唇微偏左的
不对称情况, 本文方法也能很好地将这一表情特 征迁移到目标模型上.

\section{3 联合训练的初值选取}

由于本文中联合训练的目标函数是非凸函数, 且优化方法采用了传统的梯度下降法，其初值的 选择, 这里主要是初值 $X$ 以及迭代步长 $\gamma$, 会对优 化结果产生一定影响. 为避免优化时可能陷人的 局部最优解，本文在第 3.1 节理论分析的基础上, 对多种初值组合在多组模型上进行了多次重复实 验分析, 进而选择了较优的一组作为本文联合训 练的初值. 表 3 所示为对图 7 中的 2 组模型序列应 用不同初值组合进行联合训练后的最终误差以及 运行时间的示例. 其中, 运行时间为当前初值组合 下联合训练过程所用时间，误差指优化结束后，联 合目标函数式(3)所返回的误差值; $\theta_{1}$ 和 $\theta_{2}$ 是用于 确定 $X$ 的权值 (见第 3.1 节), 步长 $\gamma$ 的取值参考了 非线性联合学习 ${ }^{[19]}$ 以及其他较常用的取值. 由于 实验中的初值组合较多, 表 3 中仅列出了部分具有 代表性的且效果较好的初值组合.

由表 3 可见, 当 $\theta_{1}=\frac{2}{4}, \theta_{2}=\frac{2}{4}$ 时, 联合目标函 数的误差在不同步长的情况下都优于另外 4 种情 
表 3 不同初值组合对联合训练误差以及时间的影响

\begin{tabular}{ccccc}
\hline$\gamma$ & $\theta_{1}$ & $\theta_{2}$ & 误差 & 运行时间 $/ \mathrm{s}$ \\
\hline \multirow{6}{*}{0.001} & $1 / 4$ & $3 / 4$ & 0.052547 & 545.041272 \\
& $2 / 4$ & $1 / 4$ & 0.055800 & 691.039410 \\
& $2 / 4$ & $2 / 4$ & 0.049886 & 572.774069 \\
& $1 / 4$ & $2 / 4$ & 0.051091 & 540.887841 \\
& $3 / 4$ & $1 / 4$ & 0.054912 & 592.488810 \\
\hline \multirow{6}{*}{0.005} & $1 / 4$ & $3 / 4$ & 0.052885 & 344.674439 \\
& $2 / 4$ & $1 / 4$ & 0.056646 & 351.732578 \\
& $2 / 4$ & $2 / 4$ & 0.052579 & 278.233437 \\
& $1 / 4$ & $2 / 4$ & 0.053832 & 335.757911 \\
& $3 / 4$ & $1 / 4$ & 0.054491 & 251.797118 \\
\hline \multirow{6}{*}{0.010} & $1 / 4$ & $3 / 4$ & 0.053352 & 150.024054 \\
& $2 / 4$ & $1 / 4$ & 0.056873 & 154.032418 \\
& $2 / 4$ & $2 / 4$ & 0.052488 & 144.811957 \\
& $1 / 4$ & $2 / 4$ & 0.053065 & 171.859157 \\
& $3 / 4$ & $1 / 4$ & 0.058803 & 153.397874 \\
\hline \multirow{6}{*}{0.020} & $1 / 4$ & $3 / 4$ & 0.058296 & 82.287067 \\
& $2 / 4$ & $1 / 4$ & 0.133783 & 425.460129 \\
& $2 / 4$ & $2 / 4$ & 0.051706 & 68.708145 \\
& $1 / 4$ & $2 / 4$ & 0.098996 & 459.555741 \\
& $3 / 4$ & $1 / 4$ & 0.056872 & 99.429762 \\
\hline \multirow{6}{*}{$*$} & & &
\end{tabular}

况，这从实验层面证明了本文将 $X$ 初值选取为 $X=\frac{1}{2} C_{1}+\frac{1}{2} C_{2}$ 可以获得较好的训练结果. 另外, 从实验结果可知, 当步长 $\gamma$ 减小时, 联合训练的误 差虽然有一定程度的降低，但误差的下降幅度在 多数情况下都不明显, 而训练时间明显延长. 在实 验过程中还发现，当步长 $\gamma \geqslant 0.02$ 时，极容易产生 无法收玫的现象. 例如, 当 $\gamma=0.02$ 时, $\theta_{1}=\frac{2}{4}$, $\theta_{2}=\frac{1}{4}$ 和 $\theta_{1}=\frac{1}{4}, \theta_{2}=\frac{2}{4}$ 这 2 种状态下都发生了未 收玫的现象，这种现象的产生符合梯度下降法中 步长偏大可能会导致发散的特性. 因此, 综合考虑 训练所用时间和优化结果, 最终设步长 $\gamma=0.01$. 本文中的其他实验均采用了类似分析过程, 最终 都表明这种初值组合可得到满意的效果. 因此, 本 文联合训练的初值设为 $X=\frac{1}{2} C_{1}+\frac{1}{2} C_{2}, \gamma=0.01$.

\section{4 性能测试}

与非线性联合学习方法 ${ }^{[19]}$ 相比, 本文方法可 以缩短联合训练的时间消耗. 分别利用 2 种方法对 图 7 图 9 中不同帧数的模型进行联合训练的时间 对比如表 4 所示.
表 42 种方法的联合训练时间

\begin{tabular}{ccrr}
\hline 模型 & 帧数 & 联合学习 ${ }^{[19]}$ & \multicolumn{1}{c}{ 本文 } \\
\hline \multirow{2}{*}{ 图 7 } & 30 & 231.802501 & 129.128155 \\
& 60 & 276.307473 & 164.670321 \\
\hline \multirow{2}{*}{ 图 8} & 30 & 668.617643 & 328.063009 \\
& 60 & 1364.321313 & 1290.578119 \\
\hline \multirow{2}{*}{ 图 9 } & 30 & 695.487182 & 412.220564 \\
& 60 & 1684.139488 & 1266.826314 \\
\hline
\end{tabular}

由表 4 可见, 本文方法在不同模型、不同帧数 下的联合训练时间都少于非线性联合学习方法的 训练时间, 且随着帧数的增加, 时间差距更加明 显. 图 8 和图 9 中模型的顶点数远多于图 7, 通过 实验可以发现，随着顶点个数的增加，时间差距也 更加明显. 可见, 本文方法有效地提高了面部表情 迁移中的训练速度.

本文方法具有一次设计、重复使用的特点，同 组序列的联合训练只需进行 1 次即可; 训练完成 后，可实现实时的表情迁移. 表 5 所示为对图 7 中 的 4 组表情迁移实验进行的性能测试, 列出了本文 方法各程序段的运行时间.

由表 5 可见，由于细节特征提取只需运行 1 次, 得到的光顺模型和细节特征向量可以反复使用, 对后续的实时表情迁移没有影响，可将该阶段作 为预处理. 预处理后, 对于单帧输人模型, 方法可 在 $1 \mathrm{~s}$ 内获得最终的具有新表情的目标模型, 因此 可达到实时的表情传递.

表 5 本文方法各程序段的运行时间

\begin{tabular}{cccc}
\hline 实验 & 细节特征提取 & 基本表情迁移 & 细节特征恢复 \\
\hline 第1组 & 0.160144 & 0.008469 & 0.951945 \\
第2组 & 0.166913 & 0.006835 & 0.954348 \\
第3 组 & 0.146852 & 0.008683 & 0.772503 \\
第4组 & 0.139187 & 0.008091 & 0.775356 \\
\hline
\end{tabular}

\section{6 结 语}

本文提出一种基于细节特征恢复的、以改进的 pDRUR 方法为核心的三维面部表情迁移方法. 细 节特征的提取与单独恢复, 克服了传统表情合成 中存在的面部个性细节特征不足的问题. pDRUR 方法的使用以及对其迭代优化函数的改进, 可大 大缩短表情合成前的联合训练时间, 有效地提高方 法的效率和稳定性的同时, 可实现实时的表情传 递, 且可在目标模型上生成高度真实感的新表情. 
本文方法在某些源模型的嘴部张合极大的情况下， 会产生目标模型的面部表情与源模型有差异的问 题，如何继续优化 nDRUR 方法，改进这种极端变 形情况下的表情传递效果是今后工作的重点.

\section{参考文献(References):}

[1] Zou Beiji, Peng Yongjin, Wu Lihua, et al. Facial expression animation based on physical model[J]. Chinese Journal of Computers, 2002, 25(3): 331-336(in Chinese)

(邹北骥，彭永进，伍立华，等. 基于物理模型的人脸表情动 画技术研究 [J]. 计算机学报, 2002, 25(3): 331-336)

[2] Dutreve L, Meyer A, Bouakaz S. Feature points based facial animation retargeting[C] //Proceedings of the ACM Symposium on Virtual Reality Software and Technology. New York: ACM Press, 2008: 197-200

[3] Chi J, Gao S S, Zhang C M. Interactive facial expression editing based on spatio-temporal coherency[J]. The Visual Computer, 2017, 33(6-8): 981-991

[4] Chuang E, Bregler C. Mood swings: Expressive speech animation[J]. ACM Transactions on Graphics, 2005, 24(2): 331-347

[5] Vlasic D, Brand M, Pfister H, et al. Face transfer with multilinear models[J]. ACM Transactions on Graphics, 2005, 24(3): $426-433$

[6] Thies J, Zollhöfer M, Nießner M, et al. Real-time expression transfer for facial reenactment $[\mathrm{J}]$. ACM Transactions on Graphics, 2015, 34(6): Article No.183

[7] Wan X M, Liu S J, Chen J X, et al. Geodesic distance-based realistic facial animation using RBF interpolation[J]. Computing in Science \& Engineering, 2012, 14(5): 49-55

[8] Feng W W, Kim B U, Yu Y Z. Real-time data driven deformation using kernel canonical correlation analysis[J]. ACM Transactions on Graphics, 2008, 27(3): 1-9

[9] Ma L, Deng Z. Real-time facial expression transformation for monocular RGB video[J]. Computer Graphics Forum, 2019, 38(1): 470-481

[10] Abrevaya V F, Wuhrer S, Boyer E. Multilinear autoencoder for 3D face model learning[C] //Proceedings of the IEEE Winter Conference on Applications of Computer Vision. Los Alamitos: IEEE Computer Society Press, 2018: 1-9

[11] Liu F, Zhu R H, Zeng D, et al. Disentangling features in 3D face shapes for joint face reconstruction and recognition[C] // Proceedings of the IEEE/CVF Conference on Computer Vision and Pattern Recognition. Los Alamitos: IEEE Computer Soci- ety Press, 2018: 5216-5225

[12] Jiang Z H, Wu Q Y, Chen K Y, et al. Disentangled representation learning for 3D face shape[C]//Proceedings of the IEEE/CVF Conference on Computer Vision and Pattern Recognition. Los Alamitos: IEEE Computer Society Press, 2019: 11949-11958

[13] Wang Hui. Researches of detail editing and symmetry of triangular meshes[D]. Dalian: Dalian University of Technology, 2013(in Chinese)

(王辉. 三角形网格细节编辑和对称的研究 $[D]$. 大连: 大连 理工大学, 2013)

[14] Miao Y W, Feng J Q, Xiao C X, et al. Detail-preserving local editing for point-sampled geometry[C]//Proceedings of the 24th Computer Graphics International Conference. Heildelberg: Springer, 2006: 673-681

[15] Wan X M, Jin X G. Data-driven facial expression synthesis via Laplacian deformation[J]. Multimedia Tools and Applications, 2012, 58(1): 109-123

[16] Xu F, Chai J X, Liu Y L, et al. Controllable high-fidelity facial performance transfer[J]. ACM Transactions on Graphics, 2014, 33(4): Article No.42

[17] Carreira-Perpiñán M Á, Lu Z D. Parametric dimensionality reduction by unsupervised regression[C]//Proceedings of the IEEE Conference on Computer Vision and Pattern Recognition. Los Alamitos: IEEE Computer Society Press, 2010: 1895-1902

[18] Taubin G. A signal processing approach to fair surface design[C] //Proceedings of the 22nd Annual Conference on Computer Graphics and Interactive Techniques. New York: ACM Press, 1995: 351-358

[19] Huang Xiaoqin, Lin Yuxu, Song Mingli, et al. 3D facial expression synthesis based on nonlinear co-learning[J]. Journal of Computer-Aided Design \& Computer Graphics, 2011, 23(2): 363-370+376(in Chinese)

(黄晓钦, 林裕旭, 宋明黎, 等. 非线性联合学习的三维人脸 表情合成方法 $[\mathrm{J}]$. 计算机辅助设计与图形学学报, 2011, 23(2): 363-370+376)

[20] Carreira-Perpinán MA, Lu Z. Dimensionality reduction by unsupervised regression[C]//Proceedings of the IEEE Conference on Computer Vision and Pattern Recognition. Los Alamitos: IEEE Computer Society Press, 2008: 1-8

[21] Noh J Y, Neumann U. Expression cloning[C] //Proceedings of the 28th Annual Conference on Computer Graphics and Interactive Techniques. New York: ACM Press, 2001: 277-288

[22] Ranjan A, Bolkart T, Sanyal S, et al. Generating 3D faces using convolutional mesh autoencoders[C] //Proceedings of the 15th European Conference on Computer Vision. Heildelberg: Springer, 2018: 725-741 